\title{
Production Planning in a Multiproduct Batch Plant under Uncertainty
}

\author{
Sebastian Engell* $^{*} \quad$ Andreas Märkert ${ }^{\dagger} \quad$ Guido Sand* \\ Rüdiger Schultz ${ }^{\dagger}$
}

\begin{abstract}
Scheduling of production plants is often affected by random side conditions such as random demand or process-inherent uncertainties. We address multiproduct batch plants where small lots of specialized products are manufactured for fast changing markets. A two-stage stochastic programming model for the production planning in a multiproduct batch plant is introduced. The model is transformed into a large-scale deterministic mixed-integer program, for which we propose a decomposition method based on Lagrangian relaxation.
\end{abstract}

2000MSC. 90C15, 90C11, 90B30, 49M27

Keywords. Stochastic Mixed-Integer Programming, Lagrangian Relaxation, Batch Processing, Production Planning, Real-Time Optimization

\section{Introduction}

Manufacturing of expandable polystyrene (EPS) in a multiproduct batch plant is considered. The production process comprises three stages: preparation, polymerization, and finishing (Figure 1 and [6]). A mixed-integer linear program (MILP) modeling this process will be presented in section 2 . The planning model we propose is part of a two-level hierarchical approach to scheduling the process. For details on the short-term scheduling we refer to [8]. Customers' demand and process-inherent factors, such as yield and

\footnotetext{
${ }^{*}$ Department of Chemical Engineering, University of Dortmund, D-44221 Dortmund, Emil-Figge-Str. 70, Germany

${ }^{\dagger}$ Department of Mathematics, Gerhardt-Mercator-University of Duisburg, D-47058 Duisburg, Lotharstr. 57, Germany
} 


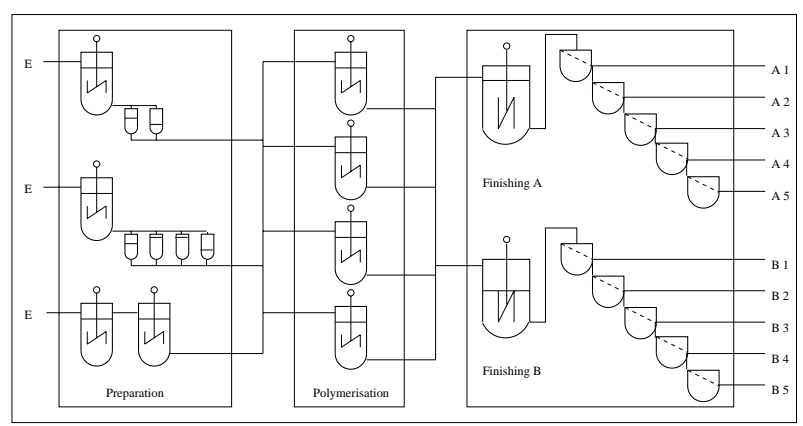

Figure 1: The EPS production process

duration of polymerizations, are typically uncertain, which motivates the extension of the MILP to a two-stage stochastic program.

Stochastic programming aims at finding non-anticipative solutions to random optimization problems [1]. In two-stage stochastic programs decisions are subdivided into 'here-and-now' decisions to be taken before and recourse decisions to be taken after the observation of the random data. Given a probability distribution of the random variable, optimization is heading for finding 'here-and-now' decisions that minimize the expectation of the total costs arising in both stages of the model. With a discrete underlying probability distribution the two-stage stochastic program can be restated as a large-scale block-angular MILP amenable to decomposition. We use the dual decomposition method developed in [3] and conclude the contribution with a short report on numerical results.

\section{Two-Stage Stochastic Programming Model}

The two-stage stochastic program is the following optimization problem, cf. [1]:

$\min _{x}\left\{c^{\mathrm{T}} x+\mathbb{E}_{\xi} \min _{y}\left\{q^{\mathrm{T}}(\omega) y\right.\right.$ s.t. $\left.\left.W(\omega) y \geq h(\omega)-T(\omega) x, y \in Y\right\}, x \in X\right\}$

where $\xi: \Omega \rightarrow \mathbb{R}^{\mathrm{m}} \times \mathbb{R}^{\mathrm{m}, \mathrm{s}} \times \mathbb{R}^{\mathrm{s}} \times \mathbb{R}^{\mathrm{n}, \mathrm{s}}, \xi(\omega)=(q(\omega), W(\omega), h(\omega), T(\omega))$ is a random variable on a probability space $(\Omega, \mathcal{A}, \mathbb{P})$, and $\mathbb{E}_{\xi}$ denotes the expectation w.r.t. the distribution of $\xi$. We distinguish first-stage $(x)$ and second-stage $(y)$ vectors in terms of their dependence on the random data. Components of both vectors may be restricted to integrality. 
For computational reasons the probability distribution of $\xi$ is assumed to be discrete or is approximated discretely. If one introduces additional second-stage vectors $y_{i}$ for each scenario $i=1, \ldots, N$, problem (1) can be transformed into the large-scale deterministic optimization problem

$$
\min _{x, y_{1}, . ., y_{N}}\left\{c^{\mathrm{T}} x+\sum_{i=1}^{N} p_{i} q_{i}^{\mathrm{T}} y_{i} \text { s.t. } T_{i} x+W_{i} y_{i} \geq h_{i}, x \in X, y_{i} \in Y, \forall i\right\},
$$

where $N$ denotes the number of mass points (scenarios) of the probability distribution of $\xi$ and $p_{i}, . ., p_{N}$ denote the scenario probabilities [3].

Single-Scenario Model The planning model has a reduced accuracy relative to the scheduling model in [8] which expresses by an aggregated time representation with equidistant time intervals and by a linear approximation of mixing effects by constant delays for each input batch. Long-term effects of the EPS production are emphasized, in particular the states of the finishing lines, which are beyond the scheduling horizon of the model in [8], are taken into account.

A single-scenario instance of the planning problem can be characterized as follows: We are given a set of EPS-types $P=\left\{1, \ldots, p^{\max }\right\}$, a set of fractions $F_{p}=\left\{1, \ldots, f_{p}^{\max }\right\}$ and recipes $R_{p}=\left\{1, \ldots, r_{p}^{\max }\right\}$ for each product $p \in P$, as well as a set of time intervals $I=\left\{1, \ldots, i^{\max }\right\}$ covering the planning horizon. We denote by $N_{i, p, r} \in \mathbb{N}$ a variable indicating the number of polymerizations in interval $i$ of type $p$ and recipe $r$ and by $x_{i, p}$ a $\{0,1\}$-variable indicating the operation state of finishing line $p$ in interval $i$. Furthermore, we introduce variables $C_{i, p} \in \mathbb{R}^{+}$for the corresponding content of the mixer tanks and parameters $B_{i, p, f} \in \mathbb{R}^{+}$specifying customers' demand.

The capacity constraints on the polymerization stage result from the number of reactors available and from the number of polymerizations running at the beginning of the corresponding time interval.

$$
\sum_{j=i}^{k} \sum_{p \in P} \sum_{r \in R} N_{j, p, r} \leq N_{i, k}^{\max } \quad \forall i \in I, \forall k \in\left\{i, \ldots, i^{\max }\right\} .
$$

The content of the mixers are constraint by a mass balance w.r.t. minimal 
and maximal feed flow $\left(G_{p}^{\min }\right.$ and $\left.G_{p}^{\max }\right)$

$$
\begin{array}{ll}
C_{i, p} \leq C_{i-1, p}+\sum_{r \in R_{p}} N_{i, p, r}-x_{i, p} G_{p}^{\min } & \forall i \in I, \forall p \in P \\
C_{i, p} \geq C_{i-1, p}+\sum_{r \in R_{p}} N_{i, p, r}-x_{i, p} G_{p}^{\max } & \forall i \in I, \forall p \in P,
\end{array}
$$

and by capacity constraints

$$
C_{i, p} \geq y_{i, p} C_{p}^{\min } \text { and } C_{i, p} \leq y_{i, p} C_{p}^{\max } \quad \forall i \in I, \forall p \in P .
$$

The binary variables $x$ enforce the feed to be 0 in off-duty intervals and the $\{0,1\}$-variables $y_{i, p}$ ensure that the mixer content equals 0 at the boundaries of idle time intervals.

$$
y_{i, p}=x_{i, p} \wedge x_{i+1, p} \quad \forall i \in I, \forall p \in P,
$$

The logical constraint (7) is transformed into three linear ones.

To guarantee a smooth operation of the finishing lines with at least $\delta \in \mathbb{N}$ subsequent off-duty and $\epsilon \in \mathbb{N}$ on-duty intervals we introduce the constraints

$$
\begin{array}{ll}
x_{i-j, p}-x_{i-j+1, p}+x_{i, p} \leq 1 & \forall i \in I, \forall p \in P, \forall j \in\{1, \ldots, \delta\} \\
x_{i-j, p}-x_{i-j+1, p}+x_{i, p} \geq 0 & \forall i \in I, \forall p \in P, \forall j \in\{1, \ldots, \epsilon\}
\end{array}
$$

The mass balance of produced and demanded polymer yield a scheme of production deficits $M_{i, p, f}^{-} \in \mathbb{R}^{+}$:

$$
\sum_{j=1}^{i} \sum_{r \in R_{p}} \rho_{p, f, r} N_{j, p, r} \geq \sum_{j=1}^{i} B_{j, p, f}-M_{i, p, f}^{-} \quad \forall i \in I, \forall p \in P, \forall f \in F_{p},
$$

where $\rho_{p, f, r}$ is the relative amount of polymer type $p$ and fraction $f$ in a polymerization batch produced according to recipe $r$.

Finally the number of start-ups and shut-downs of the finishing lines is counted.

$$
x_{i-1, p}-x_{i, p}=w_{i, p}^{+}-w_{i, p}^{-} \quad \forall i \in I, \forall p \in P
$$

The objective function models a trade-off between costs for polymerizations and operation mode changes of the finishing units on the one hand, and costs caused by production deficit (not meeting customers' requirements) on the other hand.

$$
\min _{\substack{M^{-}, N, w^{+}, w^{-}}}\left\{\sum_{\substack{i \in I \\ p \in P}}\left(\sum_{f \in F_{p}} \alpha_{i, p, f} M_{i, p, f}^{-}+\sum_{r \in R_{p}} \beta_{i, p, r} N_{i, p, r}+\gamma_{i, p}^{+} w_{i, p}^{+}+\gamma_{i, p}^{-} w_{i, p}^{-}\right)\right\}
$$


Multi-Scenario Model We propose two models (FIN, POLY) that differ w.r.t. their choices of the first-stage vector. Due to the time horizon of the scheduling model [8], information about the state of the finishing lines has to be generated by the planning model. The first approach (FIN) places - similar to the model used in [2] - accent on qualitative information about the production process, only. The variables $x_{i, p} \forall i \in\{1, . ., \hat{i}\}, \hat{i} \leq$ $i^{\text {max }}, \forall p \in P$, form the first-stage vector and a scheme of operation and idle time intervals is generated. The second model (POLY) provides information with a more quantitative character. In addition to the variables representing the finishing-line stages $\left(x_{i, p}\right)$, the polymerization variables $N_{i, p, r} \forall i \in\{1, . ., \hat{i}\}, \forall p \in P, \forall r \in R_{p}$ are included into the first-stage vector. The time interval $\hat{i} \in I$ corresponds in both cases to the length of the scheduling horizon. For further details on the interface between scheduling and planning as well as information evaluation in the scheduling model we refer to [7].

So far our numerical experiments take into account stochastic demand and stochastic polymerization yields (parameters $B_{i, p, f}$ and $\rho_{p, f, r}$ ).

\section{Dual Decomposition}

If we recall the representation (2) of a two-stage stochastic program with a discrete probability distribution, we observe that adding copies of the firststage vector transforms the program into a program with a decomposition structure.

$$
\min \left\{\sum_{j=1}^{N} p_{j}\left(c x_{j}+d_{j} y_{j}\right) \text { s.t. }\left(x_{j}, y_{j}\right) \in S^{j}, x_{1}=\ldots=x_{N}\right\}
$$

where $S^{j}=\left\{\left(x_{j}, y_{j}\right): T_{j} x_{j}+W y_{j} \geq h_{j}, x_{j} \in X, y_{j} \in Y\right\}, j=1, \ldots, N$.

The only coupling constraints are the equality constraints (non-anticipativity constraints) on the first-stage vector. A Lagrangian relaxation of the non-anticipativity constraints decomposes the program into $N$ subproblems that are similar to the MILP discussed in section 2. The resulting master problem (Lagrangian dual) is a nonlinear concave maximization.

$$
\max _{\lambda} \min \left\{\sum_{j=1}^{N} p_{j}\left(c x_{j}+d_{j} y_{j}\right)+\lambda H x_{j} \text { s.t. }\left(x_{j}, y_{j}\right) \in S^{j}, \lambda \in \mathbb{R}^{l}\right\}
$$


The matrix $H$ contains the non-anticipativity constraints. A branch-andbound algorithm successively reestablishes the equality of the components of the first-stage vector. During one branch-and-bound iteration $N$ MILP's have to be solved. For details on the used algorithm we refer to [3].

\section{Numerical Results}

The rather poor performance of a standard mixed-integer solver (Table 1) motivates the application of the problem specific decomposition algorithm. Tables 2 reports the sizes of problem instances in the structure of (13). Each

Table 1: Planning Model Solved by CPLEX in $14400 \mathrm{~s}$ of CPU-time

\begin{tabular}{lrrr}
\hline Instance $\left(i^{\max }=7, \hat{i}=3\right)$ & \multicolumn{2}{c}{ POLY } \\
No. of Scenarios & 10 & 100 \\
\hline No. of Variables & $430|566| 2,240$ & $4,030|5,606| 22,400$ \\
(integer | binary | cont.) & & & \\
No. of Constraints & 3,379 & 33,709 \\
Optimality Gaps [\%] & 10.71 & 64.49 \\
First Feasible Solution [s] & 30 & 3,200 \\
\hline
\end{tabular}

column shows the worst out of 5 runs on a SUN ULTRA 2 with a 300-MHzprocessor. We use CPLEX 7.0 to solve the subproblems and NOA3.0 [4]

Table 2: Planning Model Solved by the Decomposition Algorithm

\begin{tabular}{lrrrr}
\hline Instance $\left(i^{\max }=7, \hat{i}=3\right)$ & FIN & POLY & FIN & POLY \\
No. of Scenarios & \multicolumn{2}{c}{10} & \multicolumn{2}{c}{100} \\
\hline No. of Variables & $700|620| 2,240$ & $7,000|6,200|$ & 22,400 \\
(integer | binary | cont.) & & & & \\
No. of Constraints & 3,514 & 3,730 & 35,194 & 37,570 \\
No. of Multipliers & 54 & 324 & 594 & 3,564 \\
Solution Time [s] & 710 & 14,400 & 9,806 & 14,400 \\
Optimality Gap [\%] & 0 & 3.7 & 0 & 5.9 \\
\hline
\end{tabular}

to obtain lower bounds for the master problem. Upper bounds are generated by heuristics based on the solutions of the subproblems (frequency of occurrence, distance to average, rounding, best solution etc.).

Since neither demand nor production-yield scenarios influence the feasibility of a schedule, i.e. of a scheme of polymerizations and finishing-line 
states, each solution of a subproblem is a solution of the master problem, too. Feasible solutions for the master problem can therefore be found in the root node of the branch-and-bound tree. Problem FIN could partially be solved to optimality, since the moderate number of first-stage variables enabled us to completely enumerate.

\section{References}

[1] Birge, J.R., Louveaux, F.V.: Introduction to stochastic programming. Springer, New York (1997)

[2] Carøe, C.C., Schultz, R.: A two-stage stochastic program for unit commitment under uncertainty in a hydro-thermal power system. Schwerpunktprogramm "Echtzeit-Optimierung großer Systeme" of the Deutsche Forschungsgemeinschaft, Preprint 98-13

[3] Carøe, C.C., Schultz, R.: Dual decomposition in stochastic integer programming. Oper. Res. Lett. 23 (1999)

[4] Kiwiel, K.C.: User's guide for NOA 3.0: a Fortran package for convex nondifferentiable optimization. System Research Institute, Polish Academy of Sciences, Warsaw (1994)

[5] Sand, G.: Planungsmodell zur Echtzeitoptimierung einer verfahrenstechnischen Mehrproduktanlage bei Unsicherheit. Schwerpunktprogramm "Echtzeit-Optimierung großer Systeme" of the Deutsche Forschungsgemeinschaft, Preprint 98-28

[6] Sand, G., Engell, S., Märkert, A., Schultz, R., Schulz, C.: Approximation of an ideal online scheduler for a multiproduct batch plant. Comput. Chem. Eng. 24 (2000) 361-367

[7] Sand, G., Engell, S., Märkert, A., Schultz, R., Schulz, C.: Online Scheduling of Multiproduct Bathc Plants under Uncertainty. Proposed for: Grötschel, M., Krumke, S.O., Rambau, J. (Eds.): Online Optimization of Large Systems: State of the Art (working title), Springer, Berlin (2001)

[8] Schulz, C.: PhD thesis: Modeling and optimization of a multiproduct batch plant (in German). University of Dortmund, Dortmund (2001) (in preparation) 